\title{
Jacqueline Dreyfus, bibliothécaire et conteuse
}

Jacqueline Dreyfus, librarian and storyteller.

\section{Micheline Lebarbier}

\section{CpenEdition}

\section{Journals}

Édition électronique

URL : https://journals.openedition.org/clo/8098

DOI : $10.4000 /$ clo. 8098

ISSN : 2266-1816

\section{Éditeur}

INALCO

\section{Édition imprimée}

Date de publication : 3 décembre 2019

Pagination : 141-171

ISBN : 9782858313716

ISSN : 0396-891X

\section{Référence électronique}

Micheline Lebarbier, " Jacqueline Dreyfus, bibliothécaire et conteuse », Cahiers de littérature orale [En ligne], 86 | 2019, mis en ligne le 03 décembre 2020, consulté le 23 mars 2022. URL : http:// journals.openedition.org/clo/8098; DOI : https://doi.org/10.4000/clo.8098

\section{(c) $(1) \Theta$}

Cahiers de littérature orale est mis à disposition selon les termes de la Licence Creative Commons Attribution - Pas d'Utilisation Commerciale 4.0 International. 


\title{
Jacqueline Dreyfus, bibliothécaire et conteuse
}

\author{
Micheline LebarbieR \\ CNRS-LACITO, UMR 7107
}

\begin{abstract}
À l'heure des tweets, SMS, e-mails et autres médias, faire revivre ces « Heures du Conte » où des enfants de sept à treize ans se rassemblaient dans les locaux d'une bibliothèque enfantine, bien nommée « L'Heure joyeuse », permettra peut-être de mesurer la transformation que nos sociétés ont vécue en quelques décennies. Une heure par semaine, ils écoutaient une ou deux histoires racontées par une bibliothécaire, qui devenait conteuse pour l'occasion ${ }^{1}$.

Faire revivre quelques moments révolus de ces jeudis après-midi fait écho aux longues soirées d'hiver égayées par les histoires contées par des parents ou grands-parents dans les campagnes françaises et européennes. Il y a peu encore ces veillées étaient des lieux privilégiés de contage ${ }^{2}$, appréciées tant par les adultes que par les enfants. Ce passé oublié où la joie d'être ensemble, d'écouter la parole d'un proche, de rire ou d'être effrayés ensemble contraste radicalement avec la
\end{abstract}

1. Les archives du fonds patrimonial Heure joyeuse sont situées au sein de la médiathèque Françoise-Sagan, à Paris dans le $10^{\mathrm{e}}$ arrondissement. Que soient ici remerciées Mesdames Viviane Ezratty, Hélène Valotteau, et toutes les bibliothécaires du fonds patrimonial Heure joyeuse, pour leur accueil et leur gentillesse. Outre les documents aimablement mis à notre disposition, l'atmosphère de ce passé joyeux et studieux y est préservée avec bonheur. Les archives ont été consultées de décembre 2017 à janvier 2019. Afin d'alléger la lecture du texte, je ne mentionnerai pas à chaque fois la date de consultation.

2. Notamment dans des villages du Nord de la Roumanie, où cette tradition était encore très vivace dans les années 1970 (cf. LeBARBIER, 2011). Les personnes interrogées évoquaient ces soirées comme des moments délicieux de convivialité. 
solitude du petit écran, des tablettes et smartphones où les enfants d'aujourd'hui jouent à des jeux vidéo, naviguent sur la Toile, regardent, chacun chez soi, des séries ou des dessins animés avec parfois un frère ou une sœur, voire un parent. Être ensemble recourait, alors, à un espace géographique partagé, être ensemble aujourd'hui recourt à une connexion partagée, chacun dans un lieu différent ${ }^{3}$.

\section{Jacqueline Dreyfus, pionnière des bibliothécaires pour enfants}

Au sortir de la Première Guerre mondiale, grâce à des fonds américains, furent créés en France des espaces de culture et de lieux de vie pour permettre aux enfants qui avaient connu ces années difficiles de se reconstruire. Dès 1920, une bibliothèque publique fut créée rue Fessart dans une cité ouvrière du $19^{\mathrm{e}}$ arrondissement de Paris à l'initiative du Comité américain pour les régions dévastées. Citée comme bibliothèque «modèle », elle fut la première bibliothèque parisienne à pratiquer un libre accès et à réserver une section aux enfants; section dont la création fut l'œuvre de Jacqueline Dreyfus. À l'âge de 18 ans, elle mit en place cette petite bibliothèque enfantine en puisant dans les nombreux livres pour enfants qui provenaient de sa famille. Elle reçut également des dons de certains de ses amis.

Quant à la bibliothèque pour enfants L'Heure joyeuse, elle fut ouverte en 1924, rue Boutebrie, par Claire Huchet et Mathilde Leriche à l'initiative du Book Committee on Children's Librairies, fondation américaine dont la présidente était alors Mrs John L. Griffiths. L'Heure du conte y eut sa place dès son ouverture, animée par ces deux bibliothécaires passionnées par les contes et se définissant elles-mêmes comme ayant une âme de «baladins ». Jacqueline Dreyfus y fit plusieurs stages de 1926 à 1930, où fut appréciée sa généreuse personnalité avant d'obtenir une licence de philosophie, un diplôme supérieur de pédagogie et un diplôme de bibliothécaire, section jeunesse. Elle y travailla au catalogue et y bénéficia des leçons de Claire Huchet, qui nota son ardeur à intégrer la meilleure façon de raconter lors des séances d'Heure du conte :

Nous essayons d'apprendre à Mademoiselle Dreyfus à bien raconter des histoires. Pour cela chaque fois qu'elle raconte (c'est elle qui a l'Heure du conte des petits en ce moment), nous la

3. D'après les statistiques du logiciel de contrôle parental Qustodio, « chaque jour, les jeunes passent en moyenne quatre heures et quinze minutes sur leur ordinateur, deux heures et quinze minutes sur leur smartphone. Les samedis et dimanches sont les jours les plus chargés en connexions », ABECASsis, 2019, p. 57. 
critiquons. Nous pouvons remarquer que chaque fois elle fait des progrès sur la fois précédente, à notre avis du moins ${ }^{4}$.

Mais bibliothécaire avant tout :

Elle organise en 1928 une bibliothèque de jeunes dans une cité ouvrière d'HBM [habitations bon marché] 140 rue de Ménilmontant dans le $20^{\text {ème }}$ arrondissement. Elle prépare la future section jeunesse de la bibliothèque municipale de Périgueux (le projet n'aboutit qu'après la guerre), et celle de la rue Sorbier à Paris. Elle travaille et pratique l'Heure du conte à la bibliothèque Fessart et participe à la rédaction de la sélection de Beaux livres, Belles histoires ${ }^{5}$.

Une collection dirigée par Marguerite Gruny et Mathilde Leriche, qui animaient avec Claire Huchet la vie de la bibliothèque L'Heure joyeuse. Nommée bibliothécaire, de 1934 à 1937, à la bibliothèque de la rue Fessart, section jeunesse, forte de son expérience à L'Heure joyeuse et précédemment à la bibliothèque de Ménilmontant (1931-1932), elle s'inspira de leur fonctionnement pour l'organisation de cette bibliothèque. Elle s'inspira surtout de l'Heure du conte pour mettre en place, dans ces deux bibliothèques, des séances réservées au contage et à la lecture de récits à haute voix.

En 1939, Jacqueline Dreyfus se réfugia à Grenoble puis revint à Paris où elle fut nommée à la bibliothèque Sainte-Geneviève. Arrêtée, le 11 février 1943, pour avoir hébergé France Bloch, résistante et communiste, elle fut déportée à Auschwitz, après être passée dans différentes prisons (Fresnes, Romainville, la Conciergerie, Drancy). Elle partit vers les fours crématoires le jour même de son arrivée au camp ${ }^{6}$.

Que ces quelques pages soient un hommage à sa mémoire et au travail qu'elle mena auprès des enfants, attentive qu'elle fut à leur transmettre le meilleur de la littérature enfantine de l'époque, ainsi que les valeurs que celle-ci véhiculait. Qu'elles rendent hommage également à ces bibliothécaires-conteuses dont elle fit partie (qu'elles soient rue Boutebrie, rue Fessart, ou rue de Ménilmontant). Elles eurent à cœur tout au long de leur carrière de nourrir l'imaginaire des enfants, issus surtout des classes moyennes voire défavorisées, avec des histoires qui, en

\footnotetext{
4. Écrit Claire Huchet le 7 avril 1930 (« Cahier de Claire Huchet »).

5. Ezratty, Lévèque \& Tenier, 1994.

6. Mémoires de sa fille Dominique WeILl, Toutes mes vies, p. 74.
} 
leur apportant distraction et évasion, enrichissaient leur vie et construisaient leur personnalité.

\section{Le fonds patrimonial Heure joyeuse}

Le fonds patrimonial Heure joyeuse, outre la mémoire de toutes ces activités, recèle des documents donnés en 1945 par Raymond Weill, le mari de Jacqueline Dreyfus. Y figurent notamment les fiches, manuscrites pour la plupart, où elle consigna méticuleusement les comptes rendus de l'Heure du conte de 1934 à 1937 à la bibliothèque Fessart. Y figurent également les cahiers où elle consigna la vie de la bibliothèque de Ménilmontant pendant les années 1931-1932; les séances de prêt, les séances d'Heure du conte, les expositions qu'elle organisa, les représentations théâtrales issues des séances de lecture à haute voix de l'Heure du conte. On y trouve de nombreux commentaires sur les jeunes lecteurs, ce qu'elle connaissait de leur vie et dont elle se préoccupait. Ainsi, on retrouve à plusieurs reprises et sur différentes pages son inquiétude pour telle petite fille souffreteuse et dont elle s'enquiert auprès de la maman. Telle autre qui bégaie et dont elle s'inquiète aussi : «Elle lit énormément d'ouvrages au-dessus de son âge. Elle se rappelle de façon presque effrayante de certains livres $\gg$. Elle insiste à plusieurs reprises pour que sa maman l'emmène à la clinique. Ou sa joie au sujet de tel garçon qui devient plus ouvert lorsqu'elle lui propose de s'investir dans des activités de la bibliothèque; tel autre encore qui a réussi son certificat d'études, travaille avec son père, et qui commence à s'intéresser aux filles... Cependant, sont souvent déplorés des incidents de discipline causés par quelques garçons adolescents $^{7}$. Par exemple, on peut lire dès la première page de ce cahier :

Dès trois heures, des masses de lecteurs auxquels se joignent quelques voyous, encombrent l'escalier. [...] Nous faisons circuler (deux garçons) dans la cour avec un placard dans le dos et une cloche à la main. À quatre heures et demie nous ouvrons aux lecteurs et renvoyons les petits frères et sœurs, les mains sales, les sans papiers.

En fait, ceux-ci n'étaient pas inscrits à la bibliothèque, car les lecteurs inscrits devaient se laver les mains avant de pouvoir toucher un livre et l'emprunter.

7. Vinson dans le présent numéro, analyse ces problèmes disciplinaires comme « une manifestation de contre-culture ou de contestation culturelle » entre l'espace policé de la bibliothèque et le monde de la rue. 
Existe également dans ce fonds, sa correspondance avec diverses personnes qui sollicitaient ses conseils pour créer une bibliothèque pour enfants. J. Dreyfus se réfère toujours à l'exemple de L'Heure joyeuse à laquelle elle renvoie souvent ses interlocuteurs, tant pour l'organisation du local de la bibliothèque, que pour le choix des livres selon leur format, leur contenu et pour le système de prêt. Dans une lettre à la personne qui la consulte pour créer ce type de bibliothèque à Poitiers, elle recommande vivement qu'un stage y soit effectué, « à défaut d'école de bibliothécaire », car il est vrai que les bibliothèques d'enfants sont des « créations nouvelles ». L'Heure joyeuse reçoit tous les ans de nombreuses stagiaires désireuses de se documenter sur les bibliothèques enfantines. Elle insiste beaucoup sur leur portée sociale et éducative qui a pour but premier de développer le goût de la lecture chez l'enfant. Les activités proposées doivent être récréatives, et l'inciter à s'éloigner de distractions malsaines ; instructives, pour lui permettre de développer le goût de la recherche personnelle ; éducatives, pour qu'il puisse acquérir son sens de la responsabilité, et approfondir les matières enseignées à l'école ; et, le cas échéant, lui permettre de découvrir une orientation professionnelle pour sa vie d'adulte.

Une bibliothèque pour enfants a également une portée sociale, elle réunit des enfants de tous les milieux sans distinction de classe, toutes nationalités et de toutes les confessions. Elle invite d'ailleurs les Éclaireurs aînés de Ménilmontant à venir collaborer à la bibliothèque où ils pourraient, entre autres tâches « assurer un service régulier à l'Heure du conte des petits ». Neuf heures par semaine devraient suffire à celui qui s'engagerait pendant, par exemple, un mois pour assurer cette tâche. Elle, Jacqueline, raconterait le premier conte et l'Éclaireur le deuxième. Les qualités requises sont précisées. Outre le calme et le silence indispensables, le travail de préparation est important pour assimiler l'histoire et la réciter à haute voix avec une élocution claire et sans les « trous de mémoire » redoutés par la conteuse.

La bibliothécaire, en plus d'une connaissance approfondie de la littérature enfantine, doit avoir une formation psychologique et pédagogique (ce que Jacqueline Dreyfus possédait, nous l'avons vu plus haut). Elle pose comme un cas de conscience l'influence que la bibliothécaire peut avoir sur les enfants à travers le choix des livres.

L'éternel problème se dresse chaque fois que l'on entre en contact avec des petits : dans quelle mesure les laisser libres ? Il n'est pas question de contraindre, mais avons-nous même le droit de les influencer? 
Non seulement Jacqueline Dreyfus a bénéficié de la pratique et de l'expérience de L'Heure joyeuse, mais elle en a également retiré les mêmes principes de convivialité dans ses rapports avec les enfants. Elle se réjouit de « l'atmosphère de confiance et de gaîté » qui règne à la bibliothèque (notamment de Ménilmontant) où tous les enfants sont acceptés. Elle insiste sur « la poignée de main donnée aux lecteurs [qui] crée un important élément de cordialité dans l'ambiance de la bibliothèque ». Elle s'efforce de les responsabiliser et se réjouit de l'aide qu'ils apportent volontiers, pour ranger ou estampiller les livres. Ceci n'exclut pas les questions de discipline à faire respecter et qui reviennent de façon récurrente. Deux bibliothécaires sont indispensables, dit-elle, pour assurer la permanence et éviter le risque de vol. Elle déplore qu'un lecteur auquel elle avait refusé un prêt ait fait une tentative de vol.

Un autre incident est relaté dans le cahier de comptes rendus de la bibliothèque de Ménilmontant 1931-1932 : un billet écrit au crayon à papier, et lancé par la porte ouverte, coincé dans les lames d'un canif avec ces mots menaçants :

$\mathrm{M}^{\text {elle }}$ Jacqueline si vous rentrez chez vous c'est votre Mort ; la main noir recherché par la police depuis si ans par la rousse $[\mathrm{sic}]^{8}$.

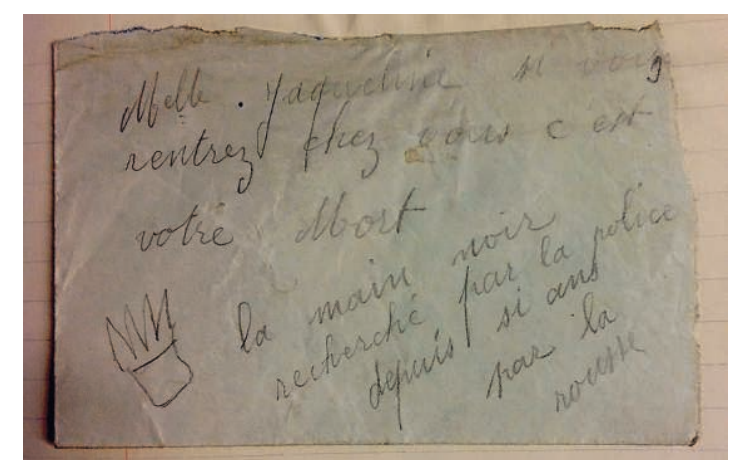

Illustration 3

« Le mot » Jacqueline Dreyfus, Comptes rendus de la bibliothèque de

Ménilmontant, 16 janvier 1932

Fonds patrimonial Heure joyeuse

Mademoiselle Jacqueline ne parut pas s'en émouvoir outre mesure, et nota : « Nous emportons le tout. Et tout est calme quand nous repartons. Mystère ! »

8. Cf. également VInson. 
J. Dreyfus participe à la constitution des fichiers de l'Heure du conte où non seulement chaque livre est recensé mais annoté avec ses appréciations concernant l'intérêt et la moralité du récit. Les réactions des enfants sont indiquées sur de nombreuses fiches tant à partir de leur lecture propre qu'à partir de leurs réactions lors des séances de contage ou de lecture à haute voix. Ainsi pour la série des Bécassine, elle souligne l'affection que les jeunes lecteurs montrent à l'endroit de « cette bonne Bécassine ».

Elle a une très haute conscience de son rôle dans le choix des livres proposés à la lecture et de sa mission pédagogique à la bibliothèque ainsi qu'à l'Heure du conte, qui est l'activité « complémentaire » la plus importante aux côtés des expositions de photos, illustrations diverses ou l'organisation des fêtes annuelles. Ainsi, elle note qu'il faut « considérer les enfants comme des individus responsables dont dépend le bon fonctionnement de la bibliothèque ». Mais surtout elle fait sienne la préoccupation commune à toutes ces bibliothécaires-conteuses :

Il ne faut pas mentir aux enfants, nous leur devons une exactitude extrême : ils doivent trouver dans ce que nous leurs racontons et dans ce que nous leur montrons pour les amuser un enseignement. Nous avons une grande responsabilité vis-à-vis d'eux.

Cet état d'esprit régnait à L'Heure joyeuse. Marguerite Gruny insiste, avec d'autres intervenants pour la jeunesse (enseignants, chefs-scouts), sur « l'importance pédagogique des auditions de contes ${ }^{9}$ ».

Certes, une bonne lecture à haute voix peut être très profitable et « mieux vaut une histoire bien lue qu'une histoire mal racontée », cependant insiste M. Gruny ${ }^{10}$ « l'histoire bien racontée est de loin préférable ». À Loulou, jeune lecteur et auditeur assidu à l'Heure du conte, Jacqueline Dreyfus demande : « Tu aimes mieux lire une histoire ou l'entendre raconter ? » à propos d'une histoire qu'il avait lue et ensuite entendue : «Oh, raconter, on y met du sien en racontant ${ }^{11}$ ! » Ce dont ne doute pas $\mathrm{M}$. Gruny ${ }^{12}$, car « le courant de sympathie

9. GRUNY, 1995, p. 13.

10. Ibid., p. 24.

11. Voir l'article de PRIVAT dans le présent numéro, l'auditeur «y met aussi du sien » par son écoute participante qui serait vue, là, comme un « contre don » à l'investissement/ don de la conteuse (cf. Mauss).

12. Ibid., p. 24. 
entre le conteur et l'auditoire est plus rapide et plus intense ${ }^{13} \gg$. Et il est évident que pour raconter, il fallait «y mettre du $\operatorname{sien}^{14} »$, et que « un conte pour être bien dit doit être bien préparé ${ }^{15} \gg$.

\section{Une oralité issue de la littérature}

Cette qualité de contage sous-entend pour les bibliothécaires-conteuses ou conteuses-bibliothécaires, selon les heures, un travail considérable de réécriture pour faire émerger une oralité cohérente à partir de la littérature écrite. De nombreux textes ont été réécrits et adaptés pour être lus ou racontés. Tout en sachant que ces récits sont pour nombre d'entre eux issus d'une oralité qui a été par la suite écrite et que (soucieuses du langage et du contenu) les conteuses-bibliothécaires ont réécrits à leur tour pour les adapter à une oralité consacrée à de jeunes auditeurs de 7 à 12 ans, voire plus jeunes encore. Immense travail de réécriture et d'adaptation !

En oralité, le sujet principal de l'énonciation est l'interprète du discours qui l'actualise à un moment donné ${ }^{16}$.

En témoignent dans les archives de L'Heure joyeuse de nombreuses pages manuscrites, souvent raturées, remaniées pour que la parole soit pleinement reçue par ce jeune public. Soulignons ici l'investissement infini de la conteuse-bibliothécaire, soucieuse de soutenir l'intérêt des enfants, dans une langue dont ils peuvent comprendre tous les termes et de leur présenter des histoires toujours édifiantes, « certes, on ne peut nier qu'il faille faire un effort

13. Par l'intermédiaire de la communication orale s'établit une interaction conteur-auditeur qui inscrit le récit dans une relation triangulaire (texte, émetteur, récepteur) et l'enrichit d'une dimension renouvelée à chaque performance. La « Parole renvoie à la dimension actionnelle du langage, comme au caractère social et culturel de toute activité langagière... », MASQUELIER, 2000, p. 25. La parole énoncée mieux que la lecture silencieuse assure ce caractère social si précieux à nos bibliothécaires, et instaure une dynamique relationnelle entre conteuse et auditoire.

14.Ibid., p. 25.

15. La performance devait être aménagée méthodiquement. Sept heures de préparation étaient nécessaires sur les neuf heures demandées aux Éclaireurs candidats à l'Heure du conte.

16. DeRIVE, 2007, p. 98. 
demandant souvent plusieurs heures pour un conte dont l'audition ne durera peut-être pas trente minutes ${ }^{17} \gg$.

Mouvement constant de balancier entre oralité et littérature, littérature et oralité, que l'on retrouve ici ${ }^{18}$.

Ainsi l'histoire du Décepteur qui circule abondamment en Europe orientale a été tirée de quatre contes, mais la lectrice-conteuse a souhaité montrer le héros « plus irréfléchi que sot » et a voulu donner à l'histoire une fin plus optimiste, car ce type de personnage est toujours montré comme étant «inguérissable ${ }^{19}$. En règle générale, on n'hésite pas à supprimer plusieurs détails « intéressants à la lecture mais ennuyeux à entendre $\gg$. Ou encore on cherchera toujours à gommer une description tragique comme par exemple, une peau qui pend du corps d'un personnage (un chien en l'occurrence), mais il ne sera pas précisé que celle-ci est ensanglantée. Ou encore une fin trop cruelle aux yeux de la conteuse sera modifiée et si une épée intervient dans le processus d'une guérison, le sang qu'elle fera couler ne sera pas mentionné. Et c'est en ce sens, notamment, que 《l'interprète » « actualise » son « discours » en fonction de son auditoire (sa tranche d'âge : les petits ou les plus grands; son genre : garçons, filles).

\section{Le catalogue établi par Jacqueline Dreyfus}

C'est avec un double objectif (lecture et audition) que Jacqueline Dreyfus mena diverses recherches dans plusieurs bibliothèques (dont la Bibliothèque nationale). Elle établit un catalogue de littérature enfantine, qui servira de vivier à l'Heure du conte, pour une lecture à haute voix ou pour une histoire contée. Il s'agit avant

17. Gruny, 1995, p. 30.

18. Et ailleurs. Des narrateurs interrogés dans la Roumanie des années 1970 révélaient leurs sources tantôt orales (parents, grands-parents, amis, voisins...), tantôt écrites (recueils de contes, almanachs...). L'effort de mémoire était sollicité de toute manière, bien que le contexte de la performance ait été autre. D'autre part, rappelons les numéros 56 (2004, Oralités et littérature. Échos, écarts, résurgences) et 62 (2007, Le livre parle. L'écrit dans la tradition orale) des Cahiers de littérature orale, où ce thème a été étudié.

19. Ce type de personnage peut, au contraire, être vu comme un sage, voire un initié qui, sous ses facéties, révèle à ceux qu'il côtoie l'étendue de leur sottise, leur incompétence ou leurs transgressions à la règle établie. Nastratin Hogea en Turquie (Boratav, 1971 ; Till l'Espiègle en Allemagne (JAnnet, 1866); Păcală en Roumanie (Lebarbier, 2011); ou encore l'Enfant Terrible en Afrique (Görög et alli). 
tout de donner aux enfants le goût de la lecture qui tient tant au cœur de toutes ces bibliothécaires-conteuses ${ }^{20}$.

« Fournir aux enfants des livres soigneusement choisis », écrit-elle dans une note de fonctionnement des bibliothèques pour enfants. Le livre doit être instructif, véhiculer un idéal et en contrepoint voir s'il peut plaire ou non aux enfants. Ce souci permanent ressort de la majorité des ouvrages recensés par Jacqueline Dreyfus. Il est spécifié si un récit est particulièrement dédié aux petits garçons, ou aux petites filles comme Mémoires d'une poupée; s'il s'agit d'une édition ancienne qui nécessite d'actualiser la langue, comme le conte Le loup galeux, qui est présenté dans une édition ancienne mais peut être « amusant à raconter en le mettant en français moderne »; enfin, si le livre peut être lu à haute voix, ou s'il s'agit d'un conte ou d'une histoire à raconter.

Elle se préoccupe également de faire correspondre les livres et les récits à l'âge de son public. Ainsi les contes mythologiques pourraient convenir aux plus âgés de ses auditeurs « formés », en les faisant précéder d'un aperçu général sur les dieux de l'Olympe. On peut, là, percevoir son souci constant d'une pédagogie qui côtoie l'aspect distrayant de la bibliothèque et de l'Heure du conte. Les animatrices de l'Heure du conte d'ailleurs collaboraient avec des instituteurs qui, souvent, rendaient visite aux bibliothèques. Mais la coopération avec les enseignants se faisait en dehors de l'école et apparaissait comme un complément ludique à l'éducation.

Plusieurs fiches sont consacrées aux histoires de Bécassine, servante au grand cœur, qui est prise comme prototype de la domestique. Les bêtises qu'elle commet sont plus le fait de l'incompréhension que de la sottise. La majorité des histoires sont issues d'un fait réel, d'une anecdote vécue. Et de noter la remarque d'un jeune Breton : « Non autonomiste mais bon Breton quand même. »

Elle visite les Éditions Languereau en février 1938, car Bécassine est devenue un succès commercial. Ses aventures ont vu le jour par hasard dans La Semaine de Suzette, une histoire en images n'ayant pas été livrée à temps. C. Languereau créa l'histoire d'une petite bonne habillée en bretonne. Au début, il s'agissait d'histoires courtes, séparées avant de devenir des albums. Jacqueline Dreyfus, lors de cet entretien, est curieuse de savoir si les enfants écrivent à l'éditeur. Oui, parfois, pour demander si Bécassine allait se marier ; ce qui est impossible car Bécassine ne vieillira jamais.

La vie d'auteurs de recueils de contes comme Andersen ou Madame d'Aulnoy est recensée, ainsi que l'origine de leurs contes. Est-ce pour présenter les auteurs

20. GrunY, 1995, p. 11. 
en même temps que leurs contes ? Plusieurs de leurs textes sont classés comme étant « à raconter » et particulièrement originaux comme La petite fille aux allumettes ou Le rossignol; ou encore nombre des contes de Madame d'Aulnoy qui décrivaient «ce qui s'était passé de remarquable en Europe » à son époque, et leurs conclusions morales, comme celle en faveur du pardon dans La princesse Rosette.

Enfin, récits et pièces de théâtre sont examinés selon plusieurs critères. Leur contenu bien sûr, s'ils sont ou non faciles à lire ou à raconter malgré, parfois, un texte « fade » ou insignifiant et un style abstrait ou « pleurnichard ». Les récits d'aventures aux descriptions scrupuleuses (de la faune et de la flore africaine ou du Pacifique, par exemple), ou historiques, ont l'avantage d'initier le jeune lecteur aux coutumes, mœurs et événements de l'époque. Notons cependant le reflet dans ces recensions de notions colonialistes, courantes dans cette période de l'entre-deux-guerres, où les populations autochtones étaient vues comme des « sauvages ».

\section{Les comptes rendus de l'Heure du conte ${ }^{21}$}

Après le prêt de livres choisis, l'Heure du conte est l'activité de médiation la plus importante de ces bibliothèques, qu'elles soient rue Boutebrie, rue Fessart ou rue de Ménilmontant.

Dans les fiches que Jacqueline Dreyfus établit de 1934 à 1937 pour l'Heure du conte à la bibliothèque Fessart, sont répertoriés avec minutie : la date; le ou les récits racontés (il y en avait souvent deux) et leur auteur ; l'édition dans laquelle il a été publié ; la durée de la séance (en général d'une demi-heure à une heure); l'assistance (le nombre de garçons et le nombre de filles) ; leur âge moyen ; la présence ou non de stagiaire (en général il y en avait une, parfois deux).

Elle signale, dans ces fiches, l'aménagement de la salle ${ }^{22}$. L'organisation spatiale laisse à désirer, au départ. Les enfants sont assis par terre, faute de place, ou sur les tables ; le manque de chaises ou de coussins lui rend la tâche difficile ; puis

21. Concernant les « pratiques rédactionnelles » des comptes rendus et fiches de l'Heure du conte, voir l'analyse de Amara dans le présent numéro.

22. À Ménilmontant, il est noté que les séances ne se tenaient pas dans la salle de lecture pour ne pas gêner les lecteurs et pouvaient avoir lieu dehors, en été, quand le temps le permettait. Le lieu des séances de contage et son aménagement ont leur importance pour que l'échange conteuse-auditoire ne soit pas « parasité par la précarité des conditions matérielles », PRIVAT, dans le présent numéro. 
une nouvelle disposition des chaises la lui facilite; ou la lampe unique qui devait dispenser une certaine pénombre afin de créer une atmosphère particulière ${ }^{23}$, propice à laisser vagabonder l'imagination. La présence de tel ou tel enfant et leurs réactions, leur assiduité ou leur absence, leur docilité ou leur indiscipline, sont commentées à chaque fois. Elle est très sensible à leur régularité, à leur appréciation, qui valident son investissement en tant que conteuse. Et elle est très fréquemment soucieuse des questions de discipline qui interfèrent, dit-elle, sur la qualité de sa performance.

Elle envoie des convocations, et placarde des affiches annonçant l'Heure du conte. Son expérience s'installe au fil des séances, pour elle et pour son auditoire, chacun se formant à l'autre, bel exemple d'interaction émetteur-récepteur, évoquée plus haut. En devenant une conteuse «formée », elle forme simultanément son auditoire. Elle se trouve alors dans une situation où est recherché un effet « d'oralité première » qui

réunit l'énonciateur et le public dans un même espace/temps, dans une situation de communication immédiate et non médiatisée qui, de ce fait, est non reproductible à l'identique ${ }^{24}$.

Elle utilise d'ailleurs le qualificatif « d'auditoire formé », lorsqu'elle déplore l'arrivée de « nouveaux » qui ne connaissent pas les règles, explicites et implicites, des séances du jeudi.

...une affiche annonçait à la porte : « L'Heure du conte est commencée. Personne ne peut plus entrer ». Certes, des retardataires sont repartis chez eux en pleurant ${ }^{25}$.

Certains jetèrent des cailloux sur la porte fermée, cassèrent un carreau et vinrent perturber les séances de lecture suivantes, déplore-t-elle dans les cahiers de la rue de Ménilmontant. Si bien qu'au début Jacqueline Dreyfus à la suite de menaces, souhaita la présence d'un agent de police, pour vite s'apercevoir que cette précaution s'avérait inutile ${ }^{26}$.

23. Voire magique, où la conteuse est nimbée d'une puissance ensorcelante aux yeux de ses jeunes auditeurs, voir Privat, ibid., p. 84.

24. BAUMgARDT \& BornAND, 2009, p. 12.

25. GRUNY, 1995, p. 43.

26. Ensuite, la discipline fut assurée par les enfants eux-mêmes. Un « chef » était nommé chaque mois et avait également en charge l'ordre matériel et la lecture aux petits. 
Par « formé », elle sous-entend, outre la discipline, l'interaction là encore, ce courant nécessaire, ce lien de confiance qui doit, à chaque fois, s'établir entre la conteuse et l'auditoire et surtout pour la conteuse elle-même. Ainsi, elle s'aperçoit qu'il est utile de distinguer les histoires pour les petits de celles pour les grands. Elle s'essaie à raconter une histoire à épisodes sur plusieurs semaines, curieuse d'observer les réactions et incertaine du succès de cette formule : «Voir quels sont ceux qui reviendront ». Et il lui arrive de raconter «impromptu » lorsque le moment semble favorable et que, surtout, « les enfants le réclament ». Dans tous les cas, affirme-t-elle, l'histoire est toujours préparée à l'avance, puisée dans des livres soigneusement choisis et renvoie à l'immense travail de préparation pour la conteuse, que la séance soit programmée ou non. Et renvoie aussi à cette notion de « conteur-scripteur » qui s'enchevêtre en l'occurrence à celle de « conteur énonciateur $\gg^{27}$.

\section{Jacqueline Dreyfus conteuse attentive...}

Mais avant tout, dans ses différents comptes rendus ${ }^{28}$, Jacqueline insiste de façon récurrente sur la qualité de sa performance, qui n'est pas nécessairement liée au succès rencontré lors de la séance « bon succès, meilleur que prévu », ou pas « conte pas tout à fait assez su ». Elle s'attarde sur l'accueil des enfants à ses différentes prestations et leurs commentaires, leurs mimiques pour prendre part au récit, singer un personnage, l'approuver ou s'en distancier ; sur leur nombre, l'interaction est différente si l'assistance est réduite et « n'inspire pas le même ton »; sur les rires, dus tantôt à l'histoire, tantôt à sa voix qu'elle s'efforce de rendre « comique ». Sont systématiquement notés leurs sentiments qui l'encouragent : «Oh, Mademoiselle raconte-le encore ! » ou « elle n'est pas assez longue ! », ou « il n'y en a pas une troisième ? [histoire] », ou encore « récriminations car il n'y a qu'une histoire ». Les exemples pourraient se multiplier à l'infini. Ou à l'inverse, leur attitude distraite ou indisciplinée, signe qu'elle n'a pas réussi à capter leur attention, du moins pour certains d'entre eux. Mais l'expression des petits visages, effrayés par les péripéties rencontrées par le héros, contractés par la laideur d'un personnage, ou encore l'attitude anxieuse de

Claire Huchet, rue Boutebrie, en revanche, eut parfois recours aux représentants de l'ordre (voir Vinson, dans le présent numéro).

27. Corinus, 2009, p. 21.

28. Qu'ils soient issus de ses fiches de la bibliothèque Fessart, ou de ses cahiers de la bibliothèque de Ménilmontant. 
ceux qui arrivent en croyant être en retard (et être renvoyés ? ou d'avoir perdu le début de l'histoire ?), est pour Jacqueline ce qui est « le plus encourageant ».

Elle est attentive au type de conte qui capte le mieux l'attention de l'auditoire. «Décidément les contes de Walhenberg leur plaisent avec ce féérique qui n'est pas enfantin », qu'elle a présentés à plusieurs reprises. Elle a conscience de l'importance de la structure du récit lorsque l'histoire n'a pas été assez travaillée pour être contée : «Les épisodes ne sont pas tout à fait assez découpés ». Et cette injonction qu'elle note comme si elle se l'adressait à elle-même : «Simplifier, simplifier », pour que les enfants ne soient pas déçus par une fin qu'ils ne saisissent pas, ou qu'ils soient insensibles à la valeur poétique d'un conte.

Ou bien, s'il arrive que le conte ne convienne pas au public en présence, garçons gouailleurs et moqueurs, peu habitués à venir, elle insistera alors sur des détails susceptibles de les faire rire, la laideur des Trois filles laides, par exemple, dans un conte de Wahlenberg.

\section{...mais trop modeste ?}

Cependant elle se remet souvent en cause, ou peut-être est-ce de son talent de conteuse dont elle doute, malgré les encouragements de Claire Huchet à L'Heure joyeuse (voir supra). Il lui arrive même de s'étonner, par exemple, du succès d'un conte d'Andersen (Le Porcher) qui offre « peu d'ouverture et d'action » et paradoxalement ne s'attribue aucunement ce succès. Même si une histoire les fait rire aux éclats, elle estime encore que leur intérêt n'était «pas violent » parce que l'histoire n'était « pas assez bien racontée ».

D'ailleurs, elle se demande « si les enfants ne sont pas là pour ne pas être autre part ? » De fait, une petite fille dite « anormale » dans les fiches, manifeste sa peur qu'il soit six heures et demie, heure à laquelle l'Heure du conte se termine, où les enfants retournent chez eux et à leur vie hors du rêve qui est leur quotidien. Ces remarques sous-tendent une préoccupation sous-jacente de la vie des enfants en dehors de la bibliothèque (préoccupations souvent évoquées dans le cahier de comptes rendus de la vie de la bibliothèque de Ménilmontant). Elle regrette qu'un public trop nombreux l'empêche de « sentir des réactions individuelles » et que ce public soit encore trop novice dans cette expérience pour que s'établisse une communion avec la conteuse, d'où son souci déjà évoqué d'être devant un « auditoire formé ». Se sentant fréquemment peu à l'aise elle-même dans cet exercice, elle souligne à ce propos que

Les Heures du Conte ne sont pas du tout ce qu'elles devraient, les enfants n'oublient pas tout autre chose pendant qu'ils écoutent 
l'histoire. Cela est sans aucun doute causé par le fait qu'il en est de même pour la conteuse ${ }^{29}$ qui « n'y croit pas assez » et ne domine pas assez son histoire (mémoire). La chose est rendue plus difficile parce que les enfants sont très nombreux et tout petits. Leur nombre plus grand transforme la chose !

« La chose ! », ainsi nomme-t-elle cet exercice à plusieurs reprises ! Et souligne qu'à ce stade de son expérience, elle avait une piètre confiance dans son talent de conteuse. Pourquoi n'y croit-elle pas assez ? Le nombre important de jeunes auditeurs impliquait-il un souci de discipline, qui revient presque comme un leitmotiv ? Peut-être lui était-il plus compliqué à gérer que dans le cadre seul de la bibliothèque ? Les retardataires à refuser, les indisciplinés à expulser. À la première lecture des fiches des années 1934-1937 (de la rue Fessart), on peut s'interroger sur l'identité de la rédactrice de certaines d'entre elles. Est-ce elle-même ou quelqu'un d'autre qui aurait assisté à la séance ? Une stagiaire, par exemple. Toutefois, l'écriture le plus souvent étant la même, et sur d'autres remarques ultérieures ainsi que sur les cahiers de la rue de Ménilmontant (années 1931-1932), on ne peut que conclure que Jacqueline, la conteuse, était assez critique envers elle-même et incriminait souvent sa mémoire ou sa préparation insuffisante ${ }^{30}$, alors que Jacqueline, la bibliothécaire, était à l'aise dans son rôle, y compris disciplinaire : demander aux enfants de ranger et de respecter les livres, d'avoir les mains propres pour les manipuler, les étiqueter à l'occasion, organiser ensemble des expositions ou des petites représentations théâtrales, fruits de l'Heure du conte et des séances de lecture.

Elle peut être gênée par un ou deux enfants plus turbulents, qui n'écoute pas, soit parce qu'il est trop petit et qu'il se roule sur le sol, soit que, tout simplement, le récit ne l'intéresse pas. Ou que le style donné à l'histoire n'ait pas été assez travaillé, signe là encore du peu de confiance en sa mémoire pour un « conte incomplètement su ». Ou encore que l'immense travail de réécriture mentionné plus haut n'ait pas été suffisamment préparé « nous ne savons pas assez l'histoire

29. Soulignons que Jacqueline parle souvent d'elle à la troisième personne ou utilise le « nous » d'auteur, et se désigne toujours par « la conteuse ».

30. Elle note là encore à l'issue d'une séance de l'Heure du conte : « Nous n'avons pas de grands "trous" mais ne possédons pourtant pas assez le récit pour lui donner toute sa portée artistique ». Préparation insuffisante à ses yeux pour qu'elle se sente être « l'interprète de son énoncé », DeRIVE, 2009, p. 99. 
pour en faire ressortir l'agrément littéraire ». Alors qu'on pourrait tout simplement penser ici que des garçons turbulents perturbaient sa performance.

...l'interprète ne reproduit pas comme un magnétophone et l'énonciation d'œuvres patrimoniales étant fondée sur la mémoire, il y a toujours une part de subjectivité qui conduit ce dernier à adapter son discours en fonction de la sensibilité et l'idéologie du moment ${ }^{31}$.

Elle semble plus à l'aise lors de séances de lecture à haute voix, où elle s'investit moins comme « interprète » du récit et qui ne sollicitent pas sa mémoire, même si le récit est long et que certains enfants partent après un épisode. Elle note l'attitude fascinée de telle petite fille, le désir de l'auditoire d'acclamer le héros avec la lectrice (en l'occurrence), partage apprécié de leur enthousiasme et d'identification avec le personnage. Mais l'Heure du conte représente pour elle une réelle épreuve, surtout quand l'assistance « est surchargée et provoque un énervement partiel des enfants et complet de la conteuse ». Elle confirme l'impression de «mal être » que provoque chez elle ces séances du jeudi.

Pour interpréter les comptes rendus avec justice, il ne faut jamais oublier que la conteuse est dans un tel état de fatigue à la fin du jeudi et du conte que celui-ci est presque toujours suivi chez elle d'une telle dépression que mémoire et jugements ne sont pas bien clairs.

Point qui explique, et atténue, ce sentiment d'insatisfaction et d'insécurité que Jacqueline manifeste lors de ses premiers comptes rendus, et qui perdure par la suite, l'accent étant souvent mis sur le lien entre la qualité de la séance et le degré de fatigue ou de préparation de la conteuse.

Cependant, elle se réjouit des réactions de son jeune public lorsqu'il est « suspendu à ses lèvres », que le silence est « religieux », et que justement, l'histoire les transporte dans un monde fabuleux. Elle note « l'atmosphère parfaitement recueillie » (c'est elle qui souligne), lorsque l'âge des auditeurs est plus homogène, tout en étant consciente, malgré le net progrès, (pour elle ? pour les enfants ?) que chacun (elle ? les enfants ?) n'arrive pas encore à s'oublier soi-même, et qu'il y « a encore pour la conteuse un grand pas à franchir ». Et lorsqu'enfin une séance est parfaitement réussie, dans un contexte plus calme et que conteuse et auditoire se sont adaptés l'un à l'autre, elle se réjouit que tout soit

31. Ibid. 
« incomparable », justement. Moins de lecteurs, même si le nombre d'auditeurs est aussi élevé, et quel bonheur que les « enfants soient délivrés d'eux-mêmes, des contingences, de la minute présente », qui est le but implicite de ces voyages imaginaires. Et que, pour cette fois, la discipline n'est pas imposée mais « intérieure » (c'est elle qui souligne). Pour préserver cette magie, elle insiste sur l'aspect drôle de l'histoire et occulte volontairement les détails qui pourraient être ressentis comme tragiques. Ou encore, lorsque

les passages parfaitement sus, donc dits, réveillent en effet l'attention de tout l'auditoire, et ce conte sentimental et moralisateur les charme grâce à la délicatesse des détails, le mystère ${ }^{32}$.

En revanche, tout est à reprendre lorsque des nouveaux arrivent. « Difficulté de satisfaire un nouveau contingent de petits garçons qui ne sont pas des auditeurs "formés" ». Ils ne savent pas comment se placer, ne sont pas « pris » par l'histoire. Ils prennent trop de temps pour s'installer et sont difficiles à satisfaire. D'où l'importance d'avoir devant elle cet auditoire « formé », où public d'habitués et conteuse se sont façonnés l'un à l'autre au fil des séances, et qui la sécurise. D’où une séance qu'elle qualifie de « succès mitigé ». Et l'année suivante, les commentaires sur l'attitude des enfants, nouveaux venus et anciens mélangés, concernent autant les différentes réactions des uns et des autres que les incertitudes de la conteuse. L'indiscipline de certains garçons qui, bien que mimant le conte, chahutent avec leur voisin ou se tiennent mal ( diables »), est « néfaste et entame l'avenir » qui deviendrait incertain des séances du jeudi. Or, malgré l'expérience qui s'affermira au cours des mois, elle portera toujours une attention particulière à la personnalité et au caractère dissipé (ou non) de ses jeunes auditeurs.

Le ton est quelque peu différent lorsque quelques rares fiches sont rédigées par une stagiaire ${ }^{33}$. L'accent n'est plus autant mis sur le silence ou les bruits que font les enfants et particulièrement leur inattention, qui, de fait, devaient être très perturbants pour la conteuse. La discipline est moins omniprésente, et la performance de la conteuse est présentée positivement. Il n'est pas question de lapsus ou d'hésitations ou de conte « mal su ou peu préparé », mais au contraire de « conte bien raconté, avec beaucoup d'intonations » qui « a tout

32. La Belle et la Bête, en l'occurrence.

33. Jacqueline note l'enthousiasme de telle stagiaire, mais rien d'étonnant dit-elle, celle-ci « n’avait jamais assisté à une Heure du conte ». 
de suite capté l'attention et l'intérêt des auditeurs ». Séances réussies dans ces cas précis ? Ou Jacqueline est-elle trop critique envers ses talents de conteuse ? Ou bien a-t-elle, dans un souci constant de perfection, conscience de séances insuffisamment préparées ? Comme pour cette histoire

qui a été plus racontée que récitée [c'est elle qui souligne]. Le texte un peu trop spirituel et trop émaillé de remarques philosophiques avait besoin d'adaptation. Dans ce cas il apparaît toujours que notre style plus plat touche beaucoup moins les enfants qu'un style poétique.

Par cette dernière remarque on pourrait entendre que Jacqueline Dreyfus ne se reconnaît par comme « conteuse », celle qui s'approprie un texte pour le faire vivre et l'interpréter à sa manière. Elle se ressentirait plutôt comme quelqu'un qui « restitue » une histoire apprise et ce, d'après ses dires, plutôt imparfaitement ${ }^{34}$. Il semblerait au fil de ses commentaires, parfois contradictoires, que ce soit le cas. Mais, le plus souvent, malgré un début de séance un peu cahoteux, le silence et l'intérêt « très vif » est dû, selon Jacqueline, au « genre d'histoire “à péripéties" avec magie »; toutefois, elle n'évoque en aucun cas son investissement personnel dans l'intérêt manifesté. Pourtant, elle note avec satisfaction qu'une personne extérieure constate leur « air heureux » au sortir de l'Heure du conte; ou les commentaires : «Elle faisait bien la voix, la demoiselle !» Et ces propos rapportés par la tante d'un jeune auditeur :

Ah ! Mademoiselle, vous avez un admirateur ! il disait à mon mari : «Ah ! je voudrais que tu l'entendes, elle raconte de ces contes, la demoiselle ! Je ne sais pas si elle les invente mais... ! »

Nombreuses appréciations extérieures qui pourraient conforter Jacqueline dans ses talents de conteuse vers une plus grande confiance. Car les conteuses fascinaient les enfants ${ }^{35}$. S'il en est fait discrètement état dans les documents consultés et au vu des doutes émis par Jacqueline Dreyfus sur ses propres prestations, on peut en déduire que les relations avec certains lecteurs-auditeurs

34. « [...] plus racontée que récitée »... n'est-ce pas là le propre d'un conteur qui devient le temps de l'énonciation «l'interprète » de son récit (Derive, 2009) ? Alors qu'elle semble le déplorer. Mais qu'entend-elle par récitée?

35. « Mademoiselle Gruny excelle à faire revivre devant ses auditeurs des pensées qu'ils ne pourraient pas comprendre en les lisant tout seuls ». Extrait d'un témoignage sur « La bibliothèque vue par ses lecteurs », GRUNY, 1995, p. 18. 
pouvaient s'apparenter à un «transfert ». «Tu auras beau dire, je sais bien que tu m'aimes mieux que les autres ».

\section{L'impact des séances sur le comportement des enfants}

Cependant, malgré son aisance incertaine en tant que conteuse et ses efforts parfois infructueux pour faire respecter une certaine discipline, Jacqueline savoure les comportements des enfants qui expriment leur résonnance avec les contes. Ainsi après avoir écouté Le bâton magique, un autre conte de Wahlenberg, elle se réjouit d'entendre à la sortie : «Qu'il faudrait bien un bâton magique pour faire la police dans l'escalier. » Ou bien, lorsqu'ils sont « pris » par le récit et que l'un deux, gouailleur, fait des remarques ironiques, ce sont les autres qui le font taire.

Et puis, malgré les retards, et l'agitation et en dépit, comme souvent, d'un conte « insuffisamment su et raconté en omettant trop de détails “jolis" », le silence se fait et les enfants sont « pris » (terme qui revient souvent). Car l'Heure du conte revêt un caractère « cérémonieux », voire « sacré » et nécessite ponctualité et régularité, constamment répétées. « Il faut le plus grand calme pour qu'une histoire ait toute sa portée. » Et lorsqu'une lecture impromptue a lieu, c'est sur la demande des enfants, même si l'atmosphère n'est pas aussi recueillie, car l'histoire racontée - nous l'avons vu - a plus d'effet sur eux que l'histoire lue, bien que toujours préparée à l'avance. Et puis, les lectures à épisodes ont un succès irrégulier, Jacqueline devant résumer les épisodes précédents pour ceux qui n'étaient pas présents aux séances précédentes; toutefois, elle note avec plaisir combien ils ont à cœur qu'aucun détail ne soit oublié. Ainsi, une souris parmi les animaux présents sur le bateau et qu'elle occultait à chaque fois dans L'histoire du Docteur Dolittle ${ }^{36}$, était systématiquement rappelée par les enfants. Ils fonctionnaient, là, comme adjuvants de l'énonciation... Cette lecture occupa quatre séances ${ }^{37}$. Une cinquantaine de pages étaient lues à chaque fois, et bien que Jacqueline dit n'avoir préparé que la première séance et qu'elle se déroula dans de « piètres conditions », le récit fut très largement apprécié. Mais le succès en fut,

\section{LOFTING.}

37. Et fit l'objet de plusieurs fiches de la fin décembre 1935. D'autres séances de lecture en occupèrent cinq, comme par exemple L'île rose de Vildrac. Là aussi, le succès fut moins important et l'auditoire moins assidu, ce qui semble dû dans tous ces cas au fait de « couper une histoire ». Furent également présentés sous cette forme des extraits d'écrits de Panaït Istrati, d'Anatole France... 
bien sûr ! dû à « Ce récit remarquable aussi par son style et par l'absence de détails inutiles $\gg$. C'est elle qui souligne. Comme si elle n'avait aucune implication dans ce succès ! Et au fait qu'il était connu par plusieurs jeunes auditeurs, qui venaient l'entendre avec plaisir. Plaisir qu'elle partagea également à le lire. Cependant ce plaisir « ne devait pas exclure la gravité indispensable aux Heures du Conte et... aux enfants ». Comme si le plaisir de conter et celui d'être apprécié par son auditoire devaient, très vite, être compensés par un immédiat rappel à l'ordre et à la gravité ! Cette gravité était, certes, nécessaire à la vocation éducative de l'Heure du conte, ainsi qu'à la conscience du « retentissement des contes sur l'esprit des enfants »; mais cette joie de conter et d'interagir avec eux, et en fonction d'eux, devait-elle être immédiatement tempérée ? D'autant que l'impact d'un récit s'avère important lorsqu'ils y font encore allusion plusieurs jours après une séance. Ou lorsqu'ils apportent des illustrations de chez eux. Gravité et plaisir sont étroitement intriqués, surtout lorsque l'intérêt se poursuit dans le temps.

Souvent les réactions de tel ou tel enfant sont nommément relatées, soit au cours de la séance : «L'auditoire est conquis à part Conchita », « Le petit Elie rit beaucoup avec son voisin ». Ou bien cette petite fille si fière d'assister à un conte pour « les grands ». La culture littéraire de certains enfants est soulignée. Tel ce garçon qui s'étonne que le conte Petit Frère et Petite Søur soit de Grimm parce que sa mère lui avait lu plusieurs contes de cet auteur, mais pas celui-là. Tel autre qui trouve que le style de Perrault est bien différent. Quant aux erreurs de la conteuse, incertaine - encore ! - dans un conte « mal su », elles sont relevées par les plus grands ${ }^{38}$. Et puis, quand le genre de l'auditoire s'affine, Jacqueline se réjouit de la présence plus nombreuse de petites filles. Elles sont des auditrices plus attentives que les garçons et plus sensibles à certains détails (couleurs des cheveux de l'héroïne entre autres éléments).

Même lorsqu'une séance s'avère au départ difficile, enfants énervés, conteuse fatiguée, le conte, le charme poétique du récit (La Reine des neiges d'Andersen, par exemple), les conquiert et les émeut. Et ces descriptions poétiques ne leur paraît jamais être « des longueurs ». Les rires sont notés, les frayeurs et les déceptions également, la joie lorsqu'ils devinent la suite de l'histoire, ou se réjouissent de la punition de l'antihéros. Comble de réactions positives devant un récit captivant, lorsque les enfants demandent à illustrer l'histoire racontée. Fait qui est à chaque fois consigné avec bonheur par Jacqueline. Car les demandes d'illustrations - ou pas - sont aussi un critère d'évaluation de l'impact d'un conte.

38. Ne deviendraient-ils pas là co-énonciateurs à certains moments de la performance ? 


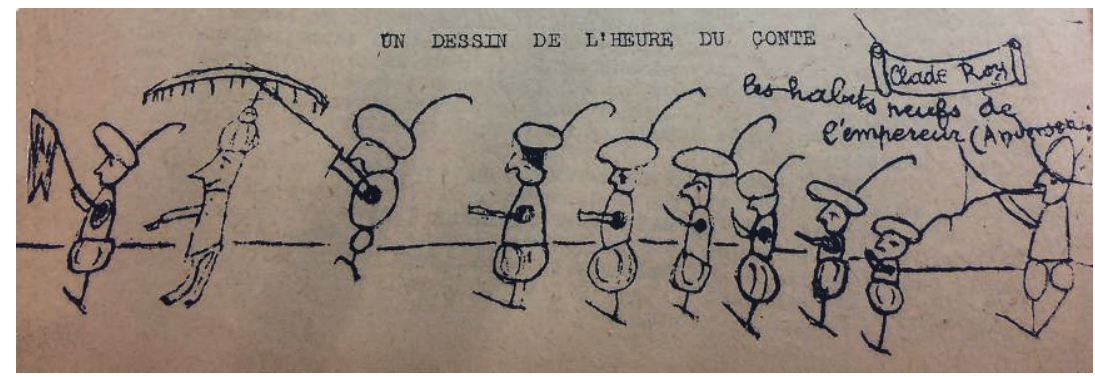

Figure 2

Dessin tiré de L'Heure Joyeuse 39 (janvier 1927)

Fonds patrimonial Heure joyeuse

Autre critère d'évaluation, les jeux de questions-réponses : « Dans quel livre? Dans quelle histoire ? Dans quel conte cet épisode ? » qui amusent beaucoup les enfants et permettent à Jacqueline de noter avec satisfaction que tous ont trouvé les bonnes réponses.

\section{Les lapsus scripturaires de Jacqueline Dreyfus}

Les doutes de la conteuse transparaissent également dans des « ratures » figurant dans ses fiches. Par exemple :

Il faut voir là le fait que la conteuse aurait pltts moins de verve pour le comique.

À la place de moins, il y a plus barré, et moins rajouté au-dessus. Est-ce une question que se pose Jacqueline ? Si oui, le lapsus scripturaire est intéressant... Rappelons qu'elle disait insister sur les détails comiques et rendre sa voix « comique ».

Autre exemple :

39. L'Heure Joyeuse, gazette des lecteurs de l'Heure joyeuse où les jeunes lecteurs pouvaient écrire des commentaires de lecture et vanter les charmes de la bibliothèque, ou à l'inverse la critiquer. Il figure d'ailleurs dans le numéro de novembre 1933, une virulente diatribe contre les bibliothécaires intitulée « De l'injustice des bibliothécaires ou Prenez garde à la... Censure ». 
Le $1^{\text {er }}$ conte est raconté avec une insuffisante fidélité au texte [...] est le sentiment ancré de la staire conteuse « Qu'il ne plaira pas $\gg$.

Les passages sont retranscrits avec les ratures et les rajouts remplaçant les mots barrés. Ils semblent significatifs de ses souhaits inexprimés et de son inconfort devant son propre jugement de ses performances. Moins au lieu de plus. Souhaiterait-elle avoir plus de verve pour le comique ? Extrême au lieu d'insuffisant. Qu'est-ce qui était extrême ? Son désir de raconter avec une extrême fidélité ? L'incertitude ? Le mal-être ? Le « mal savoir » de la conteuse, qui se ressent comme stagiaire comme au début de son activité à l'Heure du conte ? Et qui l'amène à se ressentir encore comme stagiaire et non comme la conteuse expérimentée qu'elle est devenue au fil des séances ? D'autant plus que la fiche citée a été rédigée après une année de cette expérience à la rue Fessart et fait suite à celle de la bibliothèque de Ménilmontant.

Quant à son jeune auditoire, objet de ses préoccupations, notons encore ce lapsus :

Il reste certain que les enfants maintenant moins nombreux en raison de la saison sont avides d'écouter des histoires et forment un public plus intime et moins charmant.

Voulait-elle dire, moins dissipé, moins bruyant ? Et surtout charmant, charme auquel Jacqueline laisse transparaître combien elle y est sensible.

Quant à certaines ratures, elles sont parfois énigmatiques.

Nous demandons aux lecteurs s'ils connaissent Tom Sawyer et nous teur proposons de teur raconter. Réponse négative.

Est-ce une confirmation supplémentaire de ses doutes en tant que conteuse ? Doutes qu'elle confirmera lors de la lecture de ce récit qui occupera six séances et amènera la lectrice-conteuse à se poser plusieurs questions sur l'opportunité d'histoires trop longues. Nous y reviendrons. 


\section{La langue des contes réinvestie ${ }^{40}$}

Souvent, le style poétique est souligné, ou les détails comiques, qu'elle amplifie souvent pour les amuser bien que « la conteuse aurait moins [plus en fait ?] de verve pour le comique », écrit-elle! Ou bien elle orne un repas final d'une crème au chocolat savoureuse. Même si elle adapte un texte « trop spirituel » ou « trop émaillé de remarques philosophiques », elle reste toujours consciente que son style «plus plat » touche moins son public.

...nous n'avons pas raconté en respectant assez le style si poétique et les expressions anachroniques qui donnent à ce récit toute sa saveur.

Certains noms les font rire (Panechon dans La princesse Manimala et le diamant magique $\left.{ }^{41}\right)$. D'autres passages les effraient. D'autres encore ne leur sont pas compris « la fin qui est un peu "serrée" dans ce texte et pas tout à fait assez explicite » (Le cheval enchanté dans Les contes des mille et une nuits). Un conte pour être apprécié ne doit pas être « trop descriptif » et doit se prêter à des « changements de tons », procédé très apprécié des enfants et dans lequel Jacqueline reconnaît, enfin! réussir. En revanche, elle s'interroge sur sa gestuelle et se demande si elle ne fait pas trop de gestes au cours de la narration.

Ou bien, elle déplore que « ces contes soient écrits sans soin » ou « avec négligence » à propos de Contes de Grimm (Les deux frères, Frère Joyeux, L'esprit dans la bouteille $\left.{ }^{42}\right)$. Serait-ce dû à la traduction ? et laisse supposer aussi qu'il y a plus lecture que contage et qu'en l'occurrence, Jacqueline ne s'est pas réapproprié sa langue à elle, son style à elle, mais qu'elle est restée proche de l'écriture du récit, ce que l'on déduit souvent à la lecture de ses comptes rendus.

Parfois un conte (La petite souris grise ${ }^{43}$ ) transporte tellement les enfants qu'au prénom d'une héroïne, ils se mettent à chanter et déroutent la conteuse qui s'interroge « que serait-ce si nous rendions le conte avec tous ses détails qui ont tant de charme ! » Alors, on peut se demander sur quels critères ces détails qui ont tant de charme ont été occultés. Souci de supprimer ce qui pourrait être perçu comme des «longueurs » ? Souci de maintenir une discipline qui restait toujours

40. Où la conteuse, « l'interprète... prend en charge de façon sensible la totalité du discours », DerIVE, 2009, p. 98.

41. Challendji, Sous les manguiers.

42. Contes de Grimm, Nathan.

43. Ségur, Nouveaux contes de fées, Hachette. 
fragile ? Pourtant les interactions avec son public lui sont précieuses, et son désir de le captiver est grand, tel un acteur soucieux de son succès auprès de lui. Mais de toutes ses remarques (et d'autres encore qu'il n'est pas possible de rapporter par le menu), Jacqueline ne semble pas se reconnaître comme « conteuse ${ }^{44} \gg$ à part entière, celle qui s'approprie un texte pour le faire vivre à sa manière. Elle se ressentirait plutôt comme quelqu'un qui restitue une histoire apprise et, selon elle, plutôt imparfaitement (cf. supra l'histoire «plus racontée que récitée »). Et on peut s'interroger sur le soupir de soulagement de son public enfantin lorsqu'elle annonce aux « moyens » qu'elle lira des récits au lieu de leur raconter des contes. S'agit-il, là, du genre de récits plus appréciés que des contes de fées « avec de l'or, etc. », ou bien de la lecture dans laquelle la lectrice est plus à l'aise que la conteuse ?

\section{Le choix des contes}

Jacqueline Dreyfus se préoccupe souvent de l'adéquation entre le récit et le public. Elle est très à l'écoute et sensible à la façon dont sont reçus ses contes/lectures. Si plusieurs garçons s'ennuient devant un conte plutôt réservé aux petites filles, il est décidé de réserver certaines séances aux « petits », dont les goûts et les intérêts ne sont pas encore aussi marqués. Certains contes ont une portée plus ciblée et nécessitent des précautions préalables. Ainsi pour Le silence est d'or ${ }^{45}$, la conteuse prend soin d'expliquer auparavant « qu'il ne s'agit pas de juger les Indiens dans cette histoire, peut-être ne sont-ils pas des misérables ». Cette remarque semble importante. Reflète-t-elle la morale de l'époque empreinte de notions colonisatrices, déjà notées plus haut ? Ou au contraire souhaite-t-elle mettre une distance vis-à-vis de celles-ci ? Toutefois, soulignons ce peut-être. Et lors du passage où l'héroïne a disparu, pour les enfants, elle est prisonnière des Indiens, signe de leur adhésion à l'histoire et aux conceptions de l'époque, où les Indiens sont vus comme étant les méchants.

Quant aux lectures de récits plus longs, revenons à Tom Sawyer qui eût un succès inégal. Il fut dû, en premier lieu, à son manque de préparation où, dit-elle, elle aurait pu couper des descriptions trop longues ou trop délicates « qui font traîner l'histoire pour ce public peu entraîné ». Elle coupera d'ailleurs le passage du cimetière. Il y eut aussi le manque de régularité des auditeurs qui ne reviennent pas écouter la suite de l'histoire - ce qui l'étonne - et provoque un désintérêt pour

44. « interprète » ou alors maladroite...

45. Wih, Gerbes d'histoires. 
certains passages (le rêve de Tom que tante Polly lui fait raconter, par exemple). Et puis, ce long texte à multiples péripéties ne capte pas toujours l'attention des plus petits, mais l'humour du récit les amuse (les passages dans la maison hantée, notamment). En conclusion, Jacqueline constate que l'histoire les a intéressés « et en somme convenait, cependant elle est beaucoup trop longue pour être lue une fois par semaine ». Car, en fait, l'intérêt pour les aventures de Tom Sawyer ne fut pas soutenu, en raison d'une lecture trop espacée dans le temps et aurait mieux convenu à un public mieux « formé ». Mais «ce livre constitue un bon exemple de lecture à haute voix sans coupure ». C'est elle qui souligne.

En plus de détails tragiques ou jugés scabreux et qui sont soigneusement occultés, un autre point est parfois évoqué en filigrane, le souci de la laïcité, et comment surgit au détour d'une histoire le traitement implicite donné par la conteuse. Rappelons que des enfants de toutes les confessions sont accueillis. Dans le conte Bien gardée ${ }^{46}$, Jacqueline précise qu'elle n'a pas lu la prière qui devait probablement être une prière chrétienne. Mais dans Le vieux soldat ${ }^{47}$ lorsqu' il est dit que « les sœurs vont au paradis », une petite fille reprend la conteuse. Il n'est pas précisé dans quel sens. C'est toutefois « une bonne histoire courte à raconter comme celles qui mettent le diable en jeu ». Il est vrai que, sous une forme ou une autre, le diable est présent dans toutes les religions et la notion de paradis également...

\section{L'Heure du conte, source de jeux et de représentations}

Au fil du temps, les devinettes et contes mimés, les jeux dramatiques sont devenus des activités reconnues des bibliothèques d'enfants. Des jeux de questions-réponses aussi permettaient de vérifier ce que les enfants avaient retenu des histoires lues ou contées, tout en les amusant, nous l'avons vu.

Quelques lecteurs assidus, fidèles de l'Heure du conte, furent invités à mimer certains des textes qui leur avaient plu, tel Le Rouet magique ${ }^{48}$. On rassemblait en hâte « quelques vagues accessoires ». Ou bien, afin que les enfants s'investissent plus avant dans un récit, il était procédé à des lectures dialoguées (Le Bourgeois

46. Courts récits pour les enfants et ceux qui les aiment, 1922, Bâle Georg F (suite indéchiffrable).

47. Le château imaginaire, Les livres bleus, Hachette.

48. Mirbel, Contes. 
gentilhomme) et à l'audition de la musique appropriée : le menuet éponyme de Lully ${ }^{49}$.

D'autres lectures, présentées quasi intégralement aux « grands » furent jouées/lues en courtes saynètes par des lecteurs/auditeurs chevronnés de l'Heure du conte (des « grands » là aussi, autour de 12 ans). Et les pièces de Molière s'y prêtèrent parfaitement (Les précieuses ridicules, notamment), mais les termes « amant » et « coucher contre quelqu'un » ont été coupés (censure puritaine ?). « ...l'intérêt plus soutenu vient peut-être de ce que au contraire des autres lectures » on a lu « toute la pièce ». De toute évidence, la pièce est perçue comme une histoire suivie et représentée avec plus ou moins de bonheur par les acteurs mais qui a suscité des applaudissements « tout de même ».

Quant au Malade imaginaire, s'il ne fut pas donné intégralement, il n'est pas précisé sur quels critères se sont effectuées les coupures, et les « quelques expressions anciennes [qui] ont été changées $\gg$. Mais l'intérêt fut vif et l'auditoire apprécia le côté «farce », mieux mis en valeur que le « côté "profond" » ${ }^{50}$. Et souci pédagogique exprimé de la bibliothécaire, c'est que «l'intérêt éducatif a été satisfait puisque les auditeurs paraissent vouloir emprunter l'œuvre ». Cette représentation fut suivie d'une exposition du livre Le Malade imaginaire de Félix Lorioux, qui illustra chez Hachette de nombreux livres de contes et de fables.

Et puis, Les Fourberies de Scapin, toujours réservées aux « grands », fut également source de succès, d'intérêt et de rires, où la farce « et même "guignol" » a été appréciée et la mise en scène réalisée avec les moyens du bord. Mais Jacqueline Dreyfus, là encore juge sévère d'elle-même, déplore son omission lors de la représentation : préciser que la pièce était de Molière. Toutefois, le but instructif $\mathrm{a}$, malgré cet oubli, été atteint si l'on se réfère aux réactions des uns et des autres, même les plus petits qui, au début, semblaient rebutés par un départ un peu long.

Mais il n'y eut pas que Molière qui fut source de représentation théâtrale, La princesse ensorcelée de Strowska, racontée le mardi fut jouée le vendredi avec un évident plaisir tant par les acteurs que par les figurants. Et improvisée en une semaine, La Belle au Bois Dormant fut mimée pendant la lecture du livre.

49. Dans ce cadre, est prise en compte « la dimension coopérative et interactive de l'échange verbal », MASQUELIER, 2000, p. 28.

50. Détails savoureux de cette représentation, réalisée de bric et de broc : un fauteuil a été emprunté au concierge, Toinette porte un tablier et Béline a une mantille sur la tête. 
Enfin, la lecture à haute voix avait aussi pour but d'inciter les enfants à choisir des livres qui ne figuraient pas nécessairement dans le champ de leurs intérêts.

\section{Le récit, prémisse de la lecture ou l'inverse}

Lorsqu'une lecture à haute voix a été faite sur trois ou quatre séances, le livre était exposé pour être lu sur place. Ainsi, la médiation orale, par la lecture ou le contage, incitait les enfants à lire. Et même si une lecture pouvait ne pas être appréciée par tous à cause de « passages incompréhensibles », certains, les plus grands, lisaient le livre « complètement ou partiellement » et « paraissait vraiment le goûter ».

Ou encore lorsque le public était « prêt » pour entendre un seul conte, c'est avec recueillement qu'il était écouté, et que certains enfants le relisaient « avec délice $\gg$. Et la satisfaction de ressentir ce jeune public qui «se forme » et s'ouvre à l'intérêt de la lecture est discrètement exprimée.

\section{En guise de conclusion}

Malgré ses incertitudes et ses doutes sur ses talents de conteuse Jacqueline Dreyfus eut, tout au long de ces années, à cœur de transmettre aux enfants, également par l'oralité, la richesse de la littérature écrite, qu'elle leur donnait par ailleurs à lire. Son interprétation des histoires contées offrait à ses jeunes auditeurs un autre espace imaginaire que celui que leur ouvrait la lecture. Lecture et contage leur permettaient, en complémentarité, la formation d'images qu'eux seuls généraient, interprétaient, enrichissant ainsi leur monde intérieur. Monde infiniment fertile et créateur, où les interactions avec la conteuse et les autres enfants leur ouvraient un champ d'interprétation, de partage et de communication.

C'était là un monde différent de ce que peut apporter de nos jours notre société de l'image omniprésente avec ses films, dessins animés, et vidéos de toutes sortes, où il est proposé aux enfants ${ }^{51}$ devant leurs écrans un imaginaire standardisé et formaté, où il leur est plus délicat de définir les contours de leur propre espace et d'y investir leur place et leur identité. Un monde dans lequel ils sont connectés, certes, mais isolés, où ils sont moins acteurs que spectateurs et où l'échange direct avec autrui (conteuse et auditoire) n'existe plus guère.

51. « de plus en plus éduqués par Internet », AвECAssis, 2019, p. 112. 
CAHIERS DE LITTÉRATURE ORALE

168

L'Heure du conte $-n^{\circ} 86$

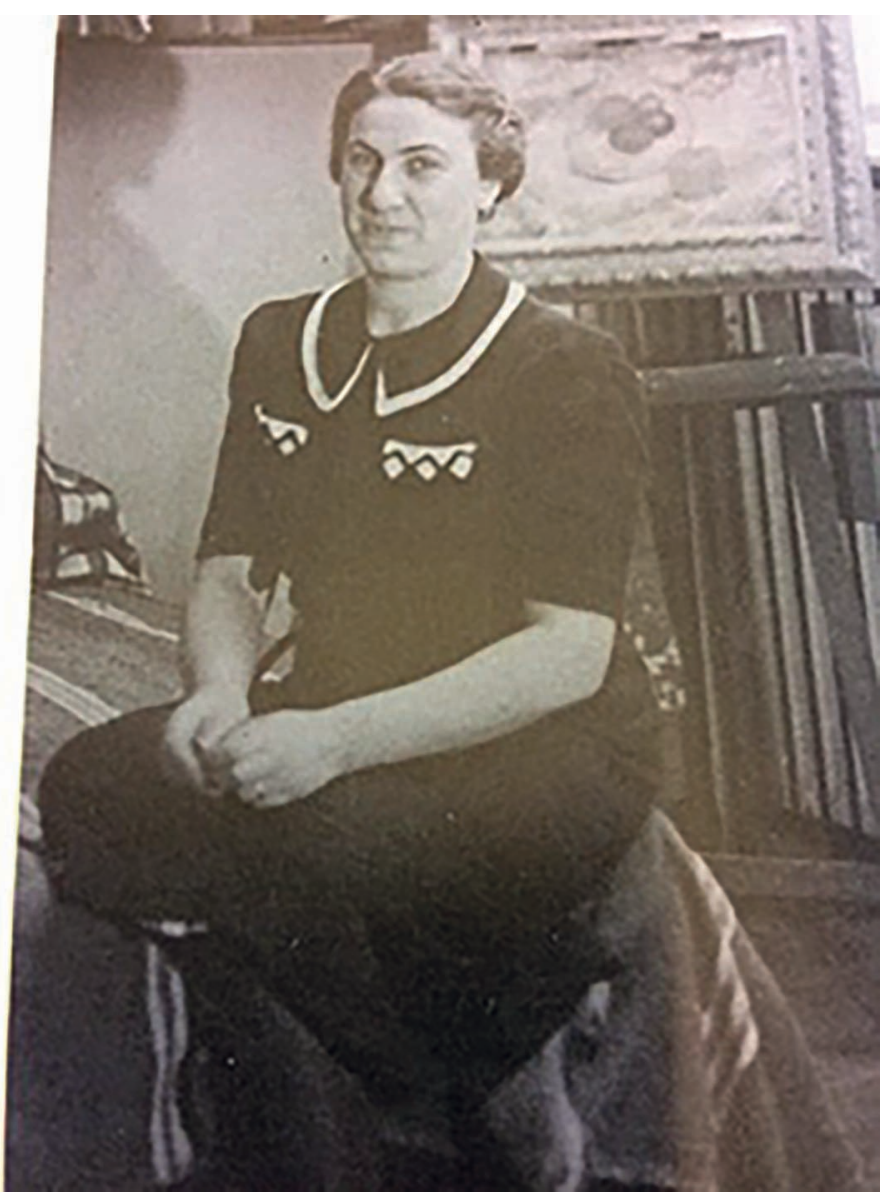

Illustration 3

Jacqueline Dreyfus

Dominique WeILL, Toutes mes vies

\section{Bibliographie}

Abecassis Éliette, 2019, L'Envie d'y croire. Journal d'une époque sans foi, Albin Michel, Paris, 207 p. 
Baumgardt Ursula \& Bornand Sandra, 2009, «Éditorial » in Cahiers de littérature orale, $\mathrm{n}^{\circ}$ 65, Publications Langue'O, Paris, p. 7-20, http://journals. openedition.org/clo/1027.

Belmont Nicole \& Privat Jean-Marie (dir.), 2007, Le Livre parle, L'écrit dans la tradition orale, Cahiers de littérature orale, $\mathrm{n}^{\circ} 62$, Publications Langues' $\mathrm{O}$, Paris, 205 p., https://doi.org/10.4000/clo.62.

Boratav Pertev 1971, Les Aires d'expansion des anecdotes de Nasreddin Hoca, Communication au $1^{\text {er }}$ Congrès international des Études balkaniques et SudEst européennes, Sofia.

Collectif, 2004, Oralité et littérature. Échos, écarts, résurgences, Cahiers de littérature orale, $\mathrm{n}^{\circ} 56$, Publications Langues'O, Paris, $284 \mathrm{p}$.

Corinus Véronique, 2009, « Félix Modock, le conteur écrivant » in Cabiers de littérature orale, n 65, Publications Langue'O, Paris, p. 21-37, https://doi. org/10.4000/clo.1086.

Derive Jean, 2009, « Des modalités de l'énonciation en littérature orale » in Cabiers de littérature orale, $\mathrm{n}^{\circ}$ 65, Publications Langue'O, Paris, p. 91-110, https://doi.org/10.4000/clo.1127.

Ezratty Viviane, Lévèque Françoise \& Tenier Françoise (dir.), 1994, 1924-1994, L'Heure Joyeuse - 70 ans de jeunesse, Médiathèque, Paris, 107 p.

Görög Veronika, Platiel Suzanne, Rey-Hulman Diana \& Seydou Christiane, 1980, Histoires d'Enfants Terribles (Afrique noire), Études et Anthologie, Préface et Conclusion par Geneviève Calame-Griaule, Maisonneuve et Larose, Paris, 301 p.

Gruny Marguerite, 1995, ABC de l'apprenti conteur. Une expérience d'« heures du conte » auprès d'enfants de 7 à 13 ans (1924-1968), Bibliothèque L'Heure joyeuse, Mairie de Paris, 165 p.

Jannet Pierre (Commentaires de), 1866, Les Aventures de Till Ulespiègle, $1^{\text {ire }}$ traduction complète faite sur l'original allemand (1519), précédé d'une notice et suivi de notes, Picard, Paris, 205 p. 
Lebarbier Micheline, 2011, Contes à rire de Roumanie. Facéties et histoires courtes, Centre de Recherche sur les Littératures et les Oralités, Karthala/ Langues O', Paris, 285 p.

Masquelier Bertrand, 2000, « Ethnographie de la parole et interlocution. Présentation » in Bertrand Masquelier \& Jean-Louis Siran (dir.), Pour une anthropologie de l'interlocution. Rhétoriques du quotidien, L'Harmattan (Logiques sociales), Paris, p. 23-56.

Mauss Marcel, 2007 [1925], Essai sur le don. Forme et raison de l'échange dans les sociétés archaïques, Presses universitaires de France (coll. Quadrige Grands textes), Paris, $248 \mathrm{p}$.

Weill Dominique, Toutes mes vies, copy media, www-copy-media.net, Canejan.

La Belle et la Bête, Contes de fées tirés de «Claude » Perrault, de Muses d'Aulnay et Leprince de Beaumont, Hachette, Paris, Bibliothèque rose ${ }^{52}$.

L'histoire du Docteur Dolittle, sa vie singulière dans son pays et ses étonnantes aventures dans les pays étrangers, Hugh Lofting, Albin Michel, Traduction Sarah Silberstein et Claire Brugell.

La Reine des neiges, Contes danois, Andersen, Édition Garnier ${ }^{53}$.

Résumé : à partir de documents conservés dans le fonds patrimonial Heure joyeuse à la médiathèque Françoise-Sagan, est exhumée la vie et l'œuvre de Jacqueline Dreyfus, qui fut l'une de ses stagiaires. Elle fut l'une des premières bibliothécaires pour enfants pendant la période de l'entre-deux-guerres et eut à cœur de leur transmettre le goût de la littérature, tant à travers la lecture qu'à travers l'audition de contes et d'histoires lues ou racontées. Formée à l'art de raconter lors des séances de l'Heure du conte à la bibliothèque L'Heure joyeuse de la rue Boutebrie, elle transporta ce « savoir conter » dans les deux bibliothèques (rue de Ménilmontant et rue Fessart) dont elle eut la charge au cours des

52. Note de J. Dreyfus : «Cette version est beaucoup plus longue et plus poétique que celle de Delagrave (Coll. Juventa) ».

53. Les livres cités par Jacqueline Dreyfus sur ses fiches et cahiers sont donnés tels que présentés dans ses documents. 
années 1931-1937. Dans ses notes et comptes rendus sont consignés la vie de ces bibliothèques, ses joies et ses doutes en tant que conteuse et ses interactions avec ses jeunes lecteurs/auditeurs. Constamment soucieuse de la transmission orale qu'elle leur offrait, souvent imparfaite à ses yeux, elle n'eut de cesse de leur présenter la quintessence de la littérature enfantine de l'époque, tant par le livre que par l'oralité. Elle disparut à Auschwitz en 1943.

Mots-clefs : bibliothèque pour enfants, littérature enfantine, conter et lire, conteuse/lectrice, auditoire, performance orale.

\section{Jacqueline Dreyfus, librarian and storyteller.}

Abstract: The story of the life and work of Jacqueline Dreyfus is brought to life from documents of the Patrimonial Collection of L'Heure joyeuse, conserved at the Françoise Sagan multimedia library in Paris. Jacqueline Dreyfus (1908-1943) had been a student-intern and later librarian at L'Heure joyeuse, an innovative movement of childrens' libraries founded after the First World War to promote the love of literature by providing access to books and to live readings and storytelling. Having herselflearned the art of storytelling at sessions of the Story Hour at L'Heure joyeuse on the rue Boutebrie, she introduced the Story Hour and the storyteller's art at the two libraries, in the rue de Menilmontant and the rue Fessart, for which she was responsible between 1931 and 1937. Her notes and reports from this period reveal the daily life of these libraries, her joys and anxieties as a performer, and her interactions with the young readers and listeners to whom she presented the best of the childrens' literature of the time, both in print and in oral performance. Jacqueline Dreyfus died at Auschwitz in 1943.

Keywords: children's library, children's literature, storytelling, oral performance, live reading.

\section{Note sur l'auteur}

Micheline Lebarbier a été chercheur au Lacito (Laboratoire de langues et civilisations à tradition orale du CNRS) et a mené des recherches sur la tradition orale en Roumanie. Elle a publié aux Éditions Karthala Contes à rire de Roumanie en 2011 et a édité Les ruses de la parole en 2017, issu d'un séminaire de recherche qu'elle anima au Lacito. Elle est membre du comité de rédaction des Cabiers de littérature orale depuis sa création en 1976. Elle y a publié de nombreux articles et coordonné plusieurs numéros. 